\title{
Consistent Network Emerge for N-Hop Network
}

\author{
Girija M.S, Thiagarajan K, Tapas Bapu B.R
}

\begin{abstract}
:
Communication is all about how you get your message across the network. Network theory is a part of grid theory in which a network can be depicted as a grid with nodes and links. Connected grid and completely connected grid representation of the network is converted to agenda reliance chart which illustrates the data dependencies and control dependencies. Fixation of the source node and sink node is made from the agenda reliance chart and the possible numbers of n-hop networks are determined through agenda reliance chart.
\end{abstract}

Index Terms: Agenda Reliance Chart, Grid, N-Hop Network, Shortest Path

\section{INTRODUCTION}

In a communication set-up, a node can send, store or receive data. In other words, any node can play the role source or sink node or intermediate node to enhance the process of communication. A hop is just a path portion between resource node and sink node [1]. The hop count refers to the number of nodes which are intermediate between the source node and sink node. There are many n-hop networks. In general, n-hop network means that there are $\mathrm{n}$ intermediate nodes between the source node and sink node. Grid theory is a study of grids which is used to model the network with nodes as vertices and paths as edges. Grid theory approach has become a critical component in the computing field including network and security [2]. Network theory [6], a study of grids has wide number of applications in many disciplines..

\section{Preliminary and Definitions}

1. Grid :

A grid [4] is an ordered pair $\mathrm{G}=(\mathrm{V}, \mathrm{E})$ where, $\mathrm{V}$ is the vertices set and $\mathrm{E}$ is the set of connections between vertices.

\section{Undirected Grid:}

An undirected grid [3] is a grid in which links have no direction i.e., the edge $(a, b)$ is identical to $(b, a)$.

\section{Directed Grid:}

A directed grid [3] is a grid in which edges have orientation. Here, edge is a set of pair of ordered vertices.

\section{Connected Grid:}

A grid is said to be connected if there is a path between every pair of vertices.

\section{Degree:}

Revised Manuscript Received on July 05, 2019

Girija M.S, Department of computer Science and Engineering, Anna University, Jeppiaar Engineering College, Chennai, India, girijamagesh@gmail.com.

Thiagarajan K, Academic Research Professor, Anna University, Jeppiaar Engineering College, Chennai, India, vidhyamannan@yahoo.com.

Tapas Bapu B.R, Department of Electronics and Communication, Anna University, S.A. Engineering, Chennai, India, tapasbapu@saec.ac.in.
The degree[5] of a vertex of the grid is the number of links incident to the vertex.

\section{Regular Grid:}

A grid [7] is said to be a regular grid if every node has the same degree.

7. Connecting Way:

Connecting way [8] is a finite or infinite sequence of links which connects a sequence of vertices.

\section{Level:}

Level is a division of the nodes into subsets that have the same distance from a given root vertex.

9. Agenda Reliance Chart (ARC):

Agenda Reliance Chart in computer science is a representation of grid that makes data dependencies and control dependencies explicit.

The organization of the paper as follows, Section II illustrates the block diagram, Section III presents the examples of 2-Regular grid, Section IV deals with 3-Regular grid, Section V presents complete grid, Section VI describes the propositions and Section VII ends up with the conclusion and future work.

\section{BLOCK Diagram OF Proposed SySTEM}

Fig. 1 illustrates the representation of the regular grid approach for N-hop networks.

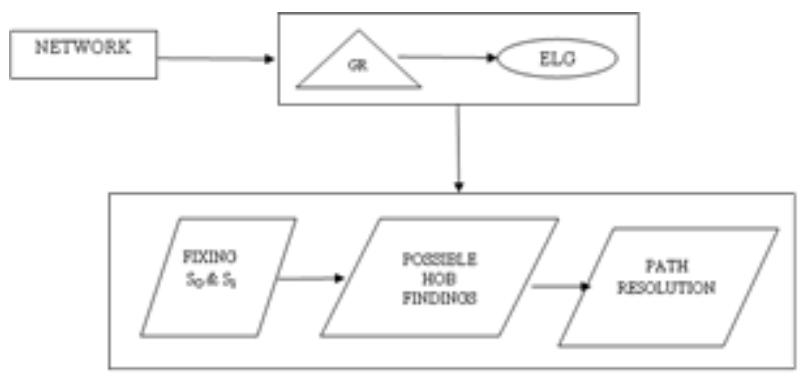

Figure 1. Block Diagram

Here,

GR - graphical representaion,

ELG- equivalent level graph ,

So \& S1 are source node and sink node. 


\section{STUDY ON POSSIBLE N -HOP NETWORK IN 2 - \\ REGULAR GRID $(\mathrm{N}>=1)$}

\section{Example: 3.1}

Consider the following grid $\mathrm{G}_{0}$ with 4 nodes and 4 links as shown below in Fig. 2.

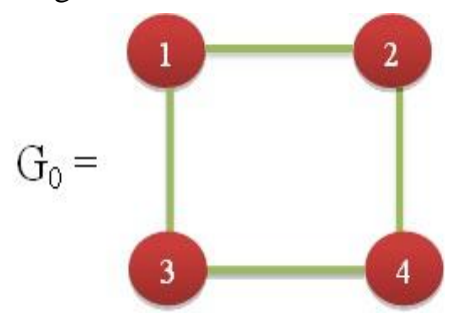

Figure 2. Grid $\mathbf{G}_{0}$

The possible 1-hop and 2-hop networks are shown in Table 1

\begin{tabular}{cc} 
Table 1. Hop Networks of $\mathbf{G}_{\mathbf{0}}$ \\
\hline 1-Hop & 2-Hop \\
\hline $1-3-4$ & $1-2-4-3$ \\
$1-2-4$ & $1-3-4-2$ \\
$2-1-3$ & $2-1-3-4$ \\
$2-4-3$ & $2-4-3-1$ \\
$3-1-2$ & $3-1-2-4$ \\
$3-4-2$ & $3-4-2-1$ \\
$4-2-1$ & $4-2-1-3$ \\
$4-3-1$ & $4-3-1-2$ \\
\hline
\end{tabular}

Corresponding Agenda Reliance Chart [5] for $\mathrm{G}_{0}$ is shown in Fig.3 (a).

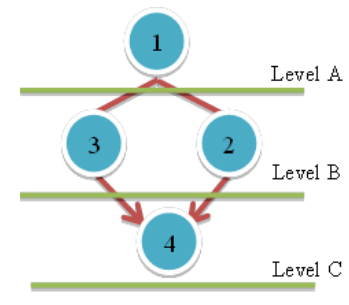

Figure 3(a). ARC of $\mathbf{G}_{0}$

Now, fixing 1 as the source node (since it is at the first level) and 4 as the sink node (because it is at the last level), the possible 1-hop networks (required level) are listed as below in Table 2(a).

Table 2(a). Required Hop Networks of G0

\begin{tabular}{ccc}
\hline Levels & Nodes & 1-Hop \\
\hline A & 1 & $1-3-4$ \\
B & 3,2 & $1-2-4$ \\
C & 4 & \\
\hline
\end{tabular}

Another way of representing corresponding Agenda Reliance Chart for $\mathrm{G}_{0}$ is shown in Fig. 3(b).



Figure 3 (b). ARC of $\mathbf{G}_{\mathbf{0}}$

Similarly, fixing 2 (as explained in Fig. 3(b) ) as the source node(since it is at the first level) and 3 as the sink node (because it is at the last level), the following Table 2(b) explains different levels of nodes [4] identified along with the 1-hop networks.

Table 2(b). Required Hop Networks of $\mathbf{G}_{\mathbf{0}}$

\begin{tabular}{ccc}
\hline Levels & Nodes & 1-Hop \\
\hline A & 2 & $2-1-3$ \\
B & 1,4 & $2-4-3$ \\
C & 3 & \\
\hline
\end{tabular}

\section{Observation:}

Here, we observed from Fig. 3(a) \& 3(b) that in 1-hop network every node of the given network can act as the source node as well as the sink node according to the fixation of the user (as user friendly).

\section{STUDY ON N-HOP NETWORK IN 3 - REgULAR GRID $(\mathbf{N}>=\mathbf{1})$}

\section{Example 4.1:}

Consider the following grid $\mathrm{G}_{1}$ with 6 nodes and 9 links as shown in Fig. 4

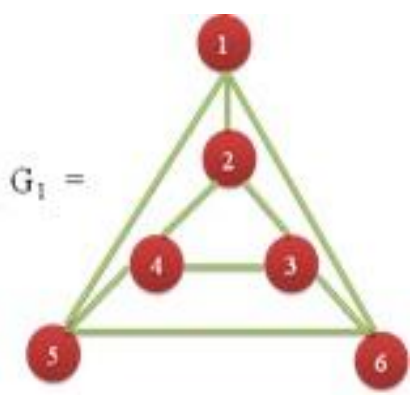

Figure 4. Grid $\mathbf{G}_{1}$

Fig. 5.shows the Agenda Reliance Chart (ARC) or level based structure of $\mathrm{G}_{1}$. 


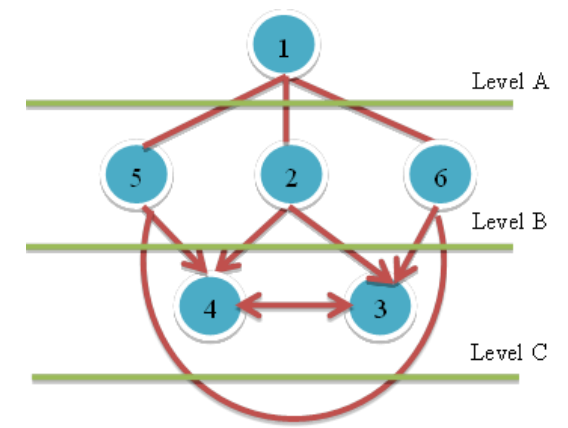

Figure 5. ARC of $\mathbf{G}_{\mathbf{1}}$

Now, fixing 1 as the source node (since it is at the first level) and $3 \& 4$ as the sink node (since it is at the last level) the possible 1-hop and 2-hop networks are as follows in Table 3.

Table 3. Required Hop Networks of $\mathbf{G}_{1}$

\begin{tabular}{cccc}
\hline Levels & Nodes & 1-Hop & 2-Hop \\
\hline A & 1 & $1-5-4$ & $1-2-4-3$ \\
B & $5,2,6$ & $1-2-4$ & $1-2-3-4$ \\
C & 4,3 & $1-2-3$ & $1-5-4-3$ \\
& & $1-6-3$ & $1-6-3-4$ \\
& & & $1-6-5-4$ \\
\hline
\end{tabular}

\section{Observation:}

If any node is fixed as the source node (since every node is having equal degree), all the other nodes can be acted as the sink node. Sink node can be reached through only 1-hop network as shown in Fig. 5, because the distance between source node and sink node is maximum 2.

\section{Example 4.2}

Consider the following grid $\mathrm{G}_{2}$ with 8 vertices and 12 edges as shown in Fig.6.

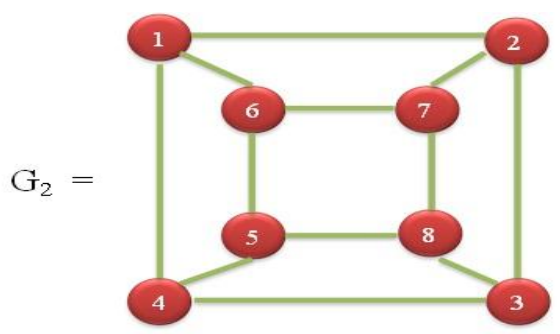

Figure 6. Grid $\mathbf{G}_{2}$

Fig. 7. Shows ARC for $\mathrm{G}_{2}$ :

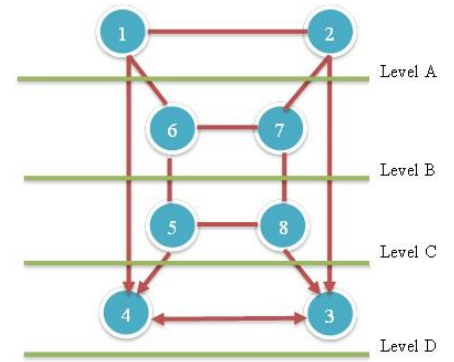

Figure 7. $A R C$ of $\mathbf{G}_{\mathbf{2}}$

Now, fixing $1 \& 2$ as the source node (since it is at the first level) and $3 \& 4$ as the sink node (since it is at the last level) the possible 1-hop, 2-hop and 3-hop networks are shown in Table 4.
Table 4. Required Hop Networks of $\mathbf{G}_{2}$

\begin{tabular}{ccccc}
\hline Levels & Nodes & 1-Hop & 2-Hop & 3-Hop \\
\hline A & 1,2 & $1-2-3$ & $1-6-5-4$ & $1-6-7-8-3$ \\
B & 6,7 & $2-1-4$ & $2-7-8-3$ & $2-7-6-5-4$ \\
C & 5,8 & & & $1-6-5-8-3$ \\
D & 3,4 & & & $2-7-8-5-4$ \\
\hline
\end{tabular}

\section{Observation:}

Here, at most 3-hop network can be constructed as shown in Table 4 after fixing up the source node and the sink node.

\section{Example 4.3}

Consider the following asymmetrical grid $\mathrm{G}_{3}$ with 10 vertices and 15 edges as shown in Fig. 8.

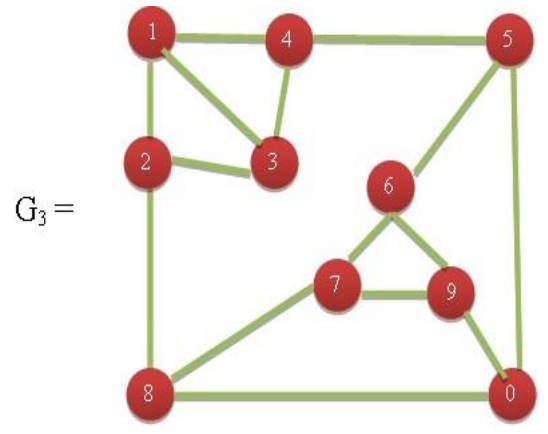

Figure 8. Grid $\mathbf{G}_{3}$

ARC of $\mathrm{G}_{3}$ is shown in Fig 9 .

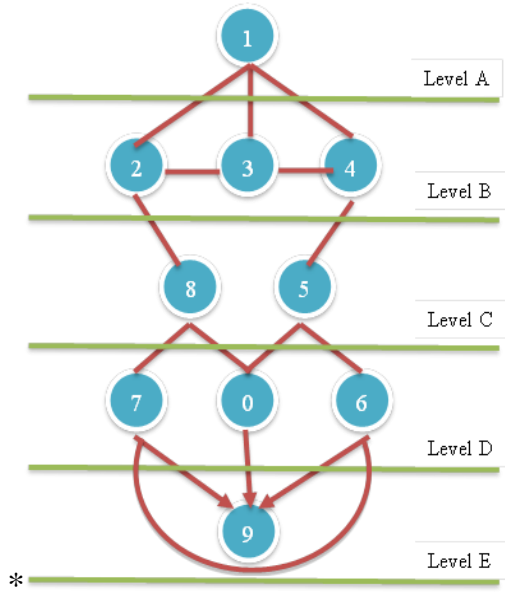

Figure 9. ARC of $\mathbf{G}_{3}$

Now, fixing 1 as the source node (since it is at the first level) and 9 as the sink node (since it is at the last level), the only possible 3 -hop networks are shown in Table 5.

Table 5. Required Hop Networks of $\mathbf{G}_{3}$

\begin{tabular}{ccc}
\hline Levels & Nodes & 3-Hop \\
\hline A & 1 & $1-2-8-7-9$ \\
B & $2,3,4$ & $1-2-8-0-9$ \\
C & 8,5 & $1-4-5-0-9$ \\
D & $7,0,6$ & $1-4-5-6-9$ \\
E & 9 & \\
\hline
\end{tabular}

\section{Observation:}

In this case 1-hop and 2-hop

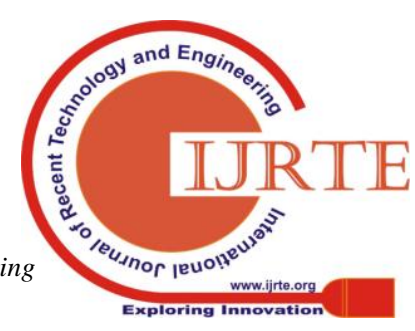


networks are not possible. But 3-hop network can be constructed as shown in Table 5.

\section{COMPLETE GRID}

Consider any regular complete grid as shown in Fig. 10. with $n$ nodes.

$\mathrm{G}_{4}$



Figure 10. Grid $\mathbf{G}_{4}$

(1) $\mathrm{ARC}$ of $\mathrm{G}_{3}$ as shown in Fig 11 shows that (n-1) number of 1-Hop will be available even though it is $n$ perfect colorable as shown in Fig. 12.

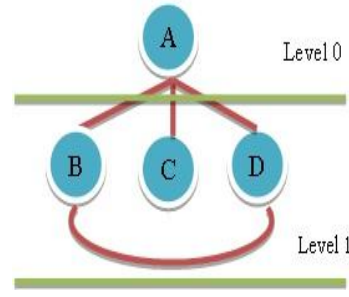

Figure 11. ARC of $\mathrm{G}_{4}$

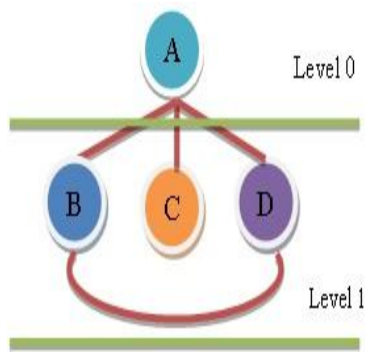

Figure 12. Grid Coloring of $\mathrm{ARC}$ of $\mathrm{G}_{\mathbf{4}}$

(2) If grid is complete regular then, ARC will have only 2 levels. The possible 1-Hop networks are shown in Table 6.

Table 6. Required Hop Networks of $\mathbf{G}_{4}$

\begin{tabular}{ccc}
\hline Levels & Nodes & 1-Hop \\
\hline 0 & A & B-A-D \\
1 & B,C,D & B-A-C \\
& & B-C-D \\
& & C-A-D \\
\hline
\end{tabular}

\section{PROPOSITIONS}

1. In a given $r-$ Regular network if there are $n$ levels in Level based program dependence grid then, at most (n-2) hop network can be constructed.

2. In a given $r$-Regular network if there are $p$ points, $q$ edges, and $\mathrm{n}$ levels with as the source node and $\mathrm{v}$ as the set of vertices, then,

$$
p \leq n * \operatorname{deg}(s) \leq \sum \operatorname{deg}(v-s)
$$

\section{CONCLUSION AND FUTURE WORK}

Regular network is taken into consideration and is converted to agenda reliance chart to identify the maximum levels of network. Source node and sink node is fixed based on the Agenda Reliance Chart and N-hop networks are determined to obtain the optimized path. The work can be extended in future by considering the ancestor networks.

\section{ACKNOWLEDGMENT}

The authors would like to express our special thanks of gratitude to Dr. Ponnammal Natarajan, Former director of Research and Development, Anna University, Chennai, India for her intuitive ideas and fruitful guidance with respect to the paper's contribution and support to complete this paper..

\section{REFERENCES}

1. Gallian J.A., "A Dynamic Survey of Graph Labeling", The Electronic journal of Combinatorics, 2015.

2. J.A. Bondy and U.S.R. Murty, "Graph Theory with Applications", London, Macmillan, 1976.

3. Harrary, F. "Graph theory", Addison Wesley, Reading Massachusetts, USA, 1969.

4. M. Chelali, L.Volkmann, "Relation Between the Lower Domination parameters and the Chromatic number of a Grid", Discrete Mathematics 274, 2004, 1-8.

5. K. Thiagarajan and P. Mansoor, "Expansion of Network through Seminode", IOSRD International Journal of Network Science, Vol 1 , Issue 1, 2017, 7-11.

6. Richard J Trudeau. "Introduction to Graph Theory“, Dover publications, 1994.

7. Béla , Bollobás, "Modern graph theory", Springer publications, 1988.

8. Gary Chartrand , Ping Zhan, "A First Course in Graph Theory",Dover publications, 2012. 